\title{
Endothelin-1-Induced Cell Hypertrophy in Cardiomyocytes is Improved by Fenofibrate: Possible Roles of Adiponectin
}

\author{
Hsu-Lung Jen ${ }^{1,5}$, Wei-Hsian Yin 1, 3,6, Jaw-Wen Chen ${ }^{2,4,6}$ and Shing-Jong Lin 2, 5, 6 \\ ${ }^{1}$ Division of Cardiology, Cheng-Hsin General Hospital, Taipei, Taiwan \\ ${ }^{2}$ Department of Medical Research and Division of Cardiology, Department of Medicine, Taipei Veterans General Hospital, Taipei, \\ Taiwan \\ ${ }^{3}$ Faculty of Medicine, National Yang-Ming University, Taipei, Taiwan \\ ${ }^{4}$ Institute of Pharmacology, National Yang-Ming University, Taipei, Taiwan \\ ${ }^{5}$ Institute of Clinical Medicine, National Yang-Ming University, Taipei, Taiwan \\ ${ }^{6}$ Cardiovascular Research Centre, School of Medicine, National Yang-Ming University, Taipei, Taiwan
}

\begin{abstract}
Aim: Previous studies demonstrated that endothelin-1 (ET-1) can significantly increase the cell size and stimulate adiponectin expression in cultured human cardiomyocytes (HCM). The aim of the present study was to investigate the effects of fenofibrate, a peroxisome proliferator-activated receptor- $\alpha$ (PPAR $\alpha)$ activator, on cell hypertrophy and adiponectin expression in vitro and in a rat model of daunorubicin-induced cardiomyopathy.

Methods: The cultured human cardiomyocytes (HCM) were stimulated with or without ET-1. The cell size and the protein expressions of PPAR $\alpha$ and adiponectin were tested by confocal Immunofluorescence study and Western blot, respectively. To study the effects of PPAR $\alpha$ activation on ET-1-induced cell hypertrophy and adiponectin protein synthesis, HCM were pretreated with fenofibrate or small interfering RNA (siRNA) of PPAR $\alpha$. Echocardiographic parameters were measured and immunohistochemistry study of myocardial adiponectin expression was conducted in the in vivo study.

Results: ET-1 significantly increased the cell size, dose-dependently suppressed the expression of $\operatorname{PPAR} \alpha$, and enhanced the expression of adiponectin; whereas, such an increase of cell size and enhancement of adiponectin expression were inhibited by the pre-treatment with fenofibrate. Addition of siRNA of PPAR $\alpha$ abolished the effects of fenofibrate. Moreover, we found that fenofibrate treatment can significantly improve the left ventricular function and reverse the myocardial expression of adiponectin.

Conclusions: Our study shows that fenofibrate may protect against ET-1-induced cardiomyocyte hypertrophy and enhanced adiponectin expression through modulation of PPAR $\alpha$ expression in vitro and limitation of daunorubicin cardiotoxicity in vivo, suggesting a novel mechanistic insight into the role of $\operatorname{PPAR} \alpha$ and adiponectin in cardiac hypertrophy and heart failure.
\end{abstract}

Key words: Peroxisome proliferator-activated receptor, Fenofibrate, Endothelin-1, Adiponectin, Cardiomyocyte hypertrophy

\section{Introduction}

Cardiac hypertrophy is considered as a compensatory mechanism that temporarily maintains pump

Address for correspondence: Wei-Hsian Yin, Division of Cardiology, Cheng-Hsin General Hospital, No. 45, Cheng-Hsin Street, Pei-Tou, Taipei 112, Taiwan, R.O.C.

E-mail: yinwh@pchome.com.tw

Received: May 1, 2016

Accepted for publication: August 9, 2016 function in response to physiological or pathological stresses. However, unremitted hypertrophy can lead to heart failure and sudden death ${ }^{1)}$. Among the neurohumoral factors activated in those conditions, endothelin-1 (ET-1) plays an important role in the genesis of myocyte hypertrophy ${ }^{2)}$.

Peroxisome proliferator-activated receptor $\alpha$ $(\operatorname{PPAR} \alpha)$ deficiency can induce impaired functional capacity of the heart ${ }^{3-6)}$. PPAR $\alpha$ activator fenofibrate can reduce cardiac hypertrophy, decrease cardiac fibro- 
sis, attenuate cardiac dysfunction, and improve survival through suppression of pro-inflammatory, pro-hypertrophic, and pro-fibrotic gene expression ${ }^{7-10}$. Fenofibrate was reported to suppress ET-1-induced cardiac hypertrophy by down-regulation of activator protein-1 (AP-1) binding capability, inhibition of p38 signaling, and modification of the nuclear factor of activated T-cells (NFAT)-related signaling systems ${ }^{11-14)}$. Moreover, atorvastatin inhibited cardiac hypertrophy through inhibition of negative cross-talk between PPAR $\alpha$ and nuclear factor-kappa B $(\mathrm{NF}-\kappa \mathrm{B})^{15)}$. Furthermore, the previous study has demonstrated that fenofibrate might also ameliorate cardiac hypertrophy via downregulation of myocardial lipid and glucose metabolism ${ }^{14)}$. However, other possible molecular mechanisms of the inhibition of cardiomyocyte hypertrophy by activated PPAR $\alpha$ remain to be elucidated.

Adiponectin, an adipokine, has also been reported to play an important role in the regulation of cardiac remodeling ${ }^{16-19)}$. Adiponectin over-expression can reverse the cardiac dysfunction induced by various pathological factors, including ET- $1^{17-19)}$. Moreover, adiponectin may significantly enhance PPAR $\alpha$ activity, and the adiponectin-dependent $\operatorname{PPAR} \alpha$ activation may play a protective role against angiotensin IIinduced cardiac hypertrophy and fibrosis ${ }^{18-20)}$. In our previous studies, both ET-1 and angiotensin II have been discovered to acutely stimulate adiponectin expression and significantly increase cell size in cultured human cardiomyocytes $(\mathrm{HCM})^{21,22)}$. As far as we know, no specific data concerning the functional relationship between fenofibrate, ET-1-induced adiponectin expression, and cell hypertrophy have yet been reported.

\section{Aim}

In the current study, we focused on surveying the effects of fenofibrate, a PPAR $\alpha$ activator, on cell hypertrophy and adiponectin expression in vitro and on amelioration of daunorubicin cardiotoxicity in a rat model. The Ethical Committee of Cheng Hsin General Hospital has approved the experiments. Throughout the study, all animals were treated in accordance with the guidelines for animal experimentation of our institute.

\section{Methods}

\section{Culture of HCM}

HCM (Cat. No. 6200) were purchased from ScienCell Research Laboratories (Carlsbad, CA) and grown in Cardiac Myocyte Medium (CMM, Cat. No. 6201) supplemented with $5 \%$ of fetal bovine serum,
$1 \%$ of cardiac myocyte growth supplement (CMGS, Cat. No. 6252), and $1 \%$ of penicillin/streptomycin solution (P/S, Cat. No. 0503). Cells were grown on poly-L-lysine-coated culture dish in a humidified incubator with $5 \% \mathrm{CO} 2$ at $37^{\circ} \mathrm{C}$. The culture medium was renewed every 3 to 4 days. With no exception, primary cells were used for experiments.

\section{Western Blot Analysis}

The cell lysate was prepared using cell lysis buffer (Cell Signaling, Beverly, MA) and then Western blot analysis was performed. The cell lysate $(25$ to $40 \mu \mathrm{g})$ was subjected to $12 \%$ sodium dodecyl sulfate-polyacrylamide gel electrophoresis and transferred to polyvinylidene difluoride membranes blotted beforehand. Having been blocked with 5\% skim milk in Tween20/PBS, blots were incubated with various primary antibodies. Equal protein loading of the samples was verified by staining monoclonal $\alpha$-tubulin or $\beta$-actin antibody (Chemicon, Temecula, CA). Blots were then incubated with the horseradish peroxidase-conjugated secondary antibodies. The signal was detected using Chemiluminescence Reagent Plus (NEN, Boston, MA). The intensity of each band was scanned and quantified using a densitometer linked to a computer software (ImageQuant, Amersham, UK).

\section{Immunocytochemical Localization of $\beta$-Actin}

Briefly, cells were cultured on glass coverslips coated with collagen IV (Sigma, Saint Louis, MO) and incubated with or without various agents. Cells were then washed with cold PBS and fixed with $4 \%$ paraformaldehyde in PBS at $4{ }^{\circ} \mathrm{C}$ for 15 mins. The cells were treated with PBS containing $0.05 \%$ Triton X-100 for 5 mins and, then, blocked with PBS containing $5 \%$ BSA at room temperature for an hour. Mouse anti-human $\beta$-actin antibody (1:400; Santa Cruz Biotechnology, Santa Cruz, CA) was added and incubated at $37^{\circ} \mathrm{C}$ for $1.5 \mathrm{hrs}$. Having been rinsed with PBS, the cells were incubated with goat anti-mouse IgG antibody (1:400; Linex Technologies, Palm Beach Gardens, FL) for one hour. After washing with PBS, the cells were mounted using Vectashield mounting medium (Vector Laboratories, Burlingame, CA) and examined with a Leica TCS SP2 confocal laser-scanning microscope (Leica Microsystems, Wetzlar, Germany).

\section{Analysis of Cardiac Myocyte Hypertrophy ${ }^{23)}$}

Phase contrast micrograph images of cardiac myocytes were captured using a camera (Nikon Europe B.V., Lijnden, Netherlands) and cell outlines were traced and digital image analyses were performed using NIS-Elements Imaging Software (Nikon Imag- 
A
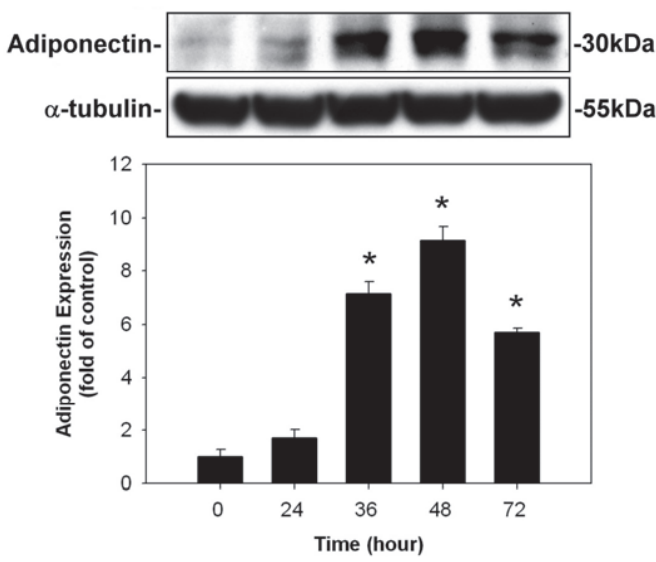

B

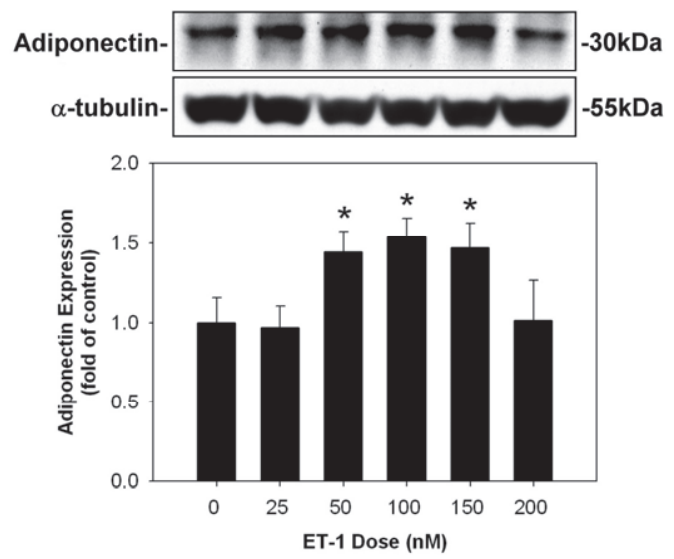

Fig. 1. Induction of adiponectin on human cardiomyocytes (HCM) by endothelin-1 (ET-1). (A) ET-1 stimulation led to an increase, reaching a peak at $48 \mathrm{hrs}$, in adiponectin protein expression, compared to those incubated with control medium. (B) ET-1 at $100 \mathrm{nM}$ ( $48 \mathrm{hrs}$ ) showed the maximal effect in enhancing adiponectin protein expression in HCM.

* denoted $P<0.05$ vs. control. Results are representative of three independent experiments.

ing, Japan). Myocytes were identified by immunofluorescence staining for $\beta$-actin. Cell surface areas calculated by planimetry were doubled to account for surfaces in contact with the plate. Cells were measured in each randomly selected field for cells treated under each condition. The identity of the samples was concealed from the observer during image analyses.

\section{Small Interfering RNA of PPAR $\alpha$}

Small interfering ribonucleic acid (siRNA), a specific double-stranded 21-nucleotide RNA sequence homologous to the target gene, was used to silence $\operatorname{PPAR} \alpha$ expression. Respectively, siRNA for PPAR $\alpha$ and Negative Control (NC) siRNA were purchased from Ambion (Austin, TX) and Santa Cruz Technology (Santa Cruz, CA). Inhibition of PPAR $\alpha$ protein expression was assessed by immunoblot analysis after transfection HCM with PPAR $\alpha-$ siRNA. Briefly, cells were grown in 100-mm dishes and transiently transfected with $20 \mathrm{nM}$ siRNA using $8 \mathrm{ml}$ of siPORT Amine (Ambion Inc., Austin, TX) in a total transfection volume of $0.5 \mathrm{ml}$ of medium. After incubation at $37^{\circ} \mathrm{C}$ in $5 \%$ CO2 for 5 hours, $1.5 \mathrm{ml}$ of normal growth medium was added and incubated with cells for 48 hours.

\section{Pharmacological Treatments with Fenofibrate}

Fenofibrate was obtained from Laboratories Fournier S.A. (Fontaine Les Dijon, France) as a gift and subsequently dissolved in dimethyl sulfoxide as a stock solution. To test the probable inhibitory effect of feno- fibrate on cardiomyocyte hypertrophy and adiponectin expression, the confluent HCM were pre-treated with the growth medium supplemented with $100 \mu \mathrm{M}$ fenofibrate for $18 \mathrm{hrs}$, followed by ET-1 $(150 \mathrm{nM})$ stimulation or control medium for $12 \mathrm{hrs}$ at $37^{\circ} \mathrm{C}$. The cardiomyocyte hypertrophy and the expression of adiponectin were next confirmed by Confocal Immunofluorescence study and Western blot, respectively. Since a daily dosage of $200 \mathrm{mg}$ of fenofibrate produced plasma concentrations within the range of $5-35 \mathrm{mg} / \mathrm{l}$ $(14-100 \mu \mathrm{M})$ in 12 dyslipidaemic patients receiving the drug over a 3-month period, the doses we studied in this experiment were clinically relevant ${ }^{24)}$.

\section{In vivo Study ${ }^{25)}$}

An in vivo study using a rat model of daunorubicin (an anthracycline)-induced cardiomyopathy was designed to support the pathophysiological relevance between fenofibrate treatment, adiponectin, and heart failure. Briefly, four-week-old male Sprague-Dawley rats were treated with a cumulative dose of $9 \mathrm{mg} / \mathrm{kg}$ body weight daunorubicin (i.v.). These animals were then randomly assigned to fenofibrate-treated (100 $\mathrm{mg} / \mathrm{kg} /$ day, p.o., the fenofibrate group, $n=6$ ) or vehicle-treated (the HF group, $n=6$ ) groups; age-matched normal rats were used as the control group $(n=6)$. Fenofibrate treatment was continued for 12 weeks. Echocardiographic parameters, such as left ventricular (LV) diastolic and systolic dimensions, and \% fractional shortening (\% FS) were measured at baseline and at the 4 th, 6 th, and 12 th weeks. The rats were 
A

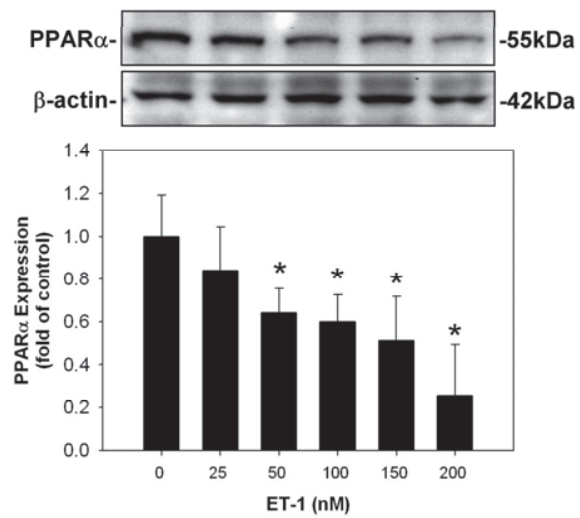

B

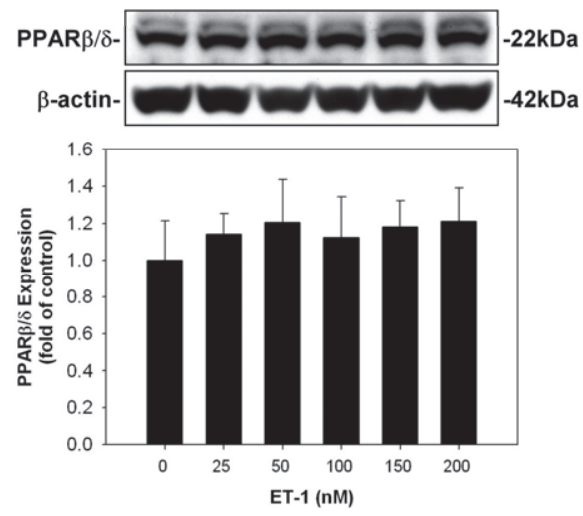

C
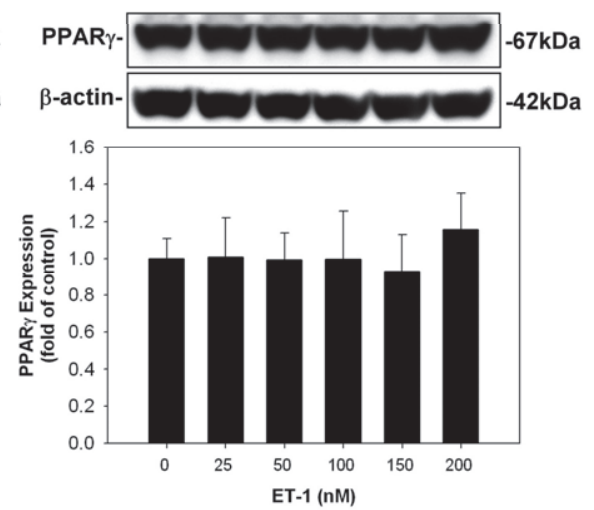

Fig. 2. Suppression of the expression of peroxisome proliferator-activated receptor $\alpha$ (PPAR $\alpha$ ) on human cardiomyocytes (HCM) by endothelin-1 (ET-1). Incubation of HCM with ET-1 dose-dependently suppressed the expression of PPAR $\alpha$ compared to those incubated with control medium; however, incubation of HCM with ET-1 did not affect the expression of PPAR $\beta / \delta$ or PPAR $y$.

* denoted $P<0.05$ vs. control. Results are representative of three independent experiments.

sacrificed in the 12th week and their blood samples were immediately collected and processed. The plasma was separated by centrifugation and then frozen under $-20^{\circ} \mathrm{C}$ and stored at that temperature until analysis. Plasma concentrations of adiponectin were measured using commercial sandwich enzyme-linked immunosorbent assays (R \& D Systems, Inc., Minneapolis, $\mathrm{MN})$.

The heart specimens were dissected from the left ventricle, and the rat myocardial tissues were fixed in $4 \%$ buffered formalin solution overnight. They were then embedded in paraffin and sectioned in $5 \mu \mathrm{m}$ thickness. The paraffin sections were de-paraffinized with xylene and stained with mouse anti-rat adiponectin antibodies (R\&D Systems, Inc. Minneapolis, MN) overnight. After being washed in PBS, the sections were incubated for 1 hour at room temperature with horseradish peroxidase-conjugated rabbit anti-mouse IgG. Next, $0.1 \%$ DAB was mixed in Tris $\mathrm{HCl}$ and $\mathrm{H}_{2} \mathrm{O}_{2}$, and the mixture was dropped onto the slides; after 3-5 minutes, the stained tissues were observed with a microscope. Finally, the sections were washed with PBS again, counter-stained with Hematoxylin, mounted using mounting medium, and then examined with a microscope.

\section{Statistics Analyses}

All values were expressed as mean \pm SEM. Comparisons between multiple groups were determined by means of a one-way analysis of variance (ANOVA) followed by Dunnett's test. A $P$ value $<0.05$ was considered statistically significant.

\section{Results}

\section{Induction of Adiponectin on HCM by ET-1}

The ET-1-induced adiponectin protein expressions were both time- and dose-dependent. ET-1 stimulation led to an increase in adiponectin protein expression (reaching a peak at 48 hrs.), compared to those incubated with control medium (Fig. 1A). Moreover, ET-1 at $100 \mathrm{nM}$ showed the maximal effect in enhancing adiponectin protein expression in HCM; hence, the concentration of ET-1 used for the following experiments was $100 \mathrm{nM}$ for 48 hrs (Fig. 1B).

\section{Suppression of the Expression of PPAR $\alpha$ on HCM by ET-1}

Incubation of HCM with ET-1 dose-dependently suppressed the expression of $\operatorname{PPAR} \alpha$ compared to those incubated with control medium (Fig. 2); however, incubation of HCM with ET-1 did not affect the expression of $\operatorname{PPAR} \beta / \delta$ or $\operatorname{PPAR} \gamma$.

\section{Enhancement of Adiponectin Expression by ET-1 in HCM was Inhibited by Pre-Treatment with Fenofibrate}

HCM were pre-treated with fenofibrate (a PPAR $\alpha$ activator) at various indicated concentration levels, 30 mins before the addition of control medium and with $100 \mathrm{nM}$ ET-1 for 48 hrs. The enhanced adiponectin protein expression in ET-1-stimulated HCM was significantly reduced dose-dependently, after the HCM had been pretreated with the growth medium supplemented with fenofibrate (Fig. 3A). Addition of siRNA 
A

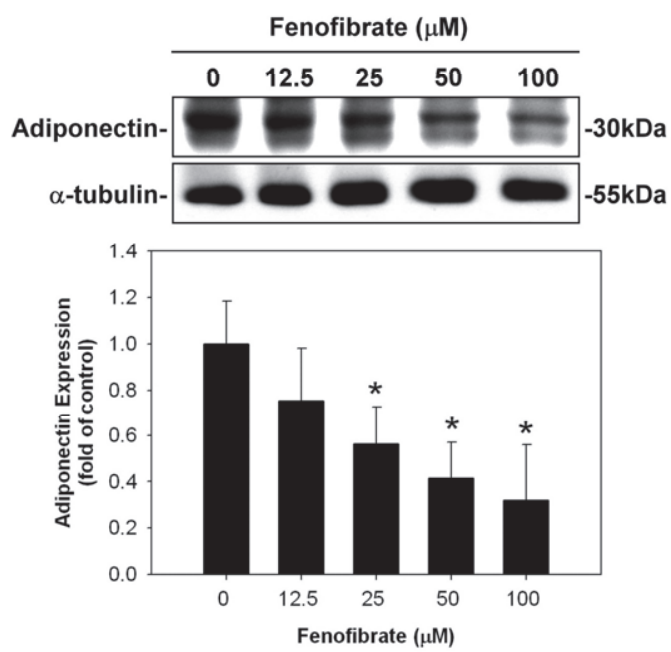

B

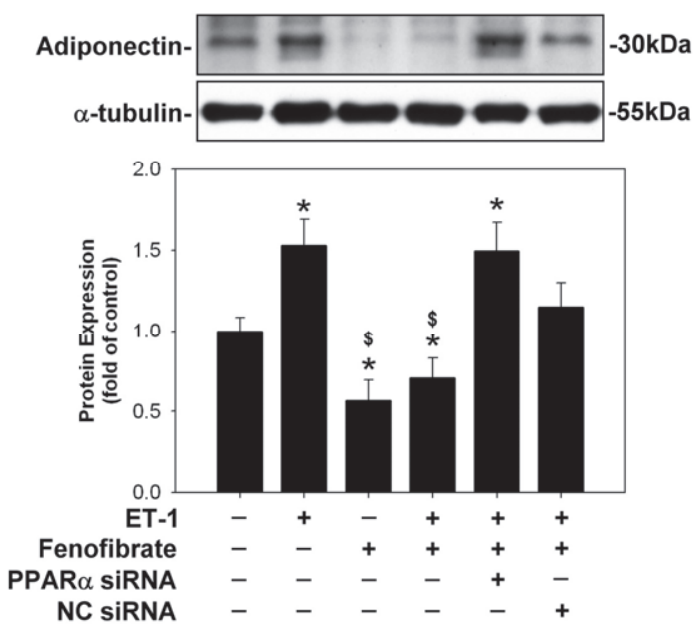

Fig. 3. Enhancement of adiponectin expression by endothelin-1 (ET-1) in human cardiomyocytes (HCM) was inhibited by pre-treatment with fenofibrate. (A) Enhanced adiponectin protein expression in ET-1-stimulated HCM was significantly reduced dose-dependently, after the HCM had been pretreated with the growth medium supplemented with fenofibrate. (B) Addition of small interfering RNA (siRNA) of peroxisome proliferator-activated receptor $\alpha(\operatorname{PPAR} \alpha)$ abolished the effects of fenofibrate.

* denoted $P<0.05$ vs. control. Results are representative of three independent experiments.

of PPAR $\alpha$ abolished the effects of fenofibrate (Fig. 3B).

\section{ET-1 Induced Hypertrophy of Cardiomyocytes in vitro; Whereas, Such Increase of Cell Size was} Inhibited by Pre-Treatment with Fenofibrate (Fig. 4)

To examine the effects of ET- 1 in cardiomyocytes at the cellular level, we used in vitro cell culture studying cardiomyocyte hypertrophy with HCM. To assess cellular hypertrophy, the cell size of cardiomyocytes was observed using confocal immunofluorescence study. All cells were pretreated with fenofibrate 100 $\mu \mathrm{M}$ for 30 mins and, subsequently, stimulated with 100 nM ET-1 for 48 hrs. ET-1 significantly increased the cell size of cardiomyocytes (upper right panel), compared to that of the control group (upper left panel); but fenofibrate inhibited ET-1-induced cellular hypertrophy (lower left panel). In addition, the small interfering RNA (siRNA) of peroxisome proliferator-activated receptor $\alpha(\operatorname{PPAR} \alpha)$, instead of the negative control (NC) siRNA, abolished the effects of fenofibrate (lower middle and right panels).

\section{Results of the in vivo Study (Table 1 and Fig. 5)}

In the in vivo study, heart weight/body weight (HW/BW) was significantly increased in HF group $(P<0.05$ vs. Control group) and was reduced by feno- fibrate treatment (as shown in Table 1), although the reduction did not reach statistical significance. Compared with those of the control group, circulating levels of adiponectin were significantly increased in HF group and fenofibrate group $(P<0.05)$. Moreover, they were significantly lower in fenofibrate group vs. HF group $(P<0.05)$.

Fig. 5A and 5B show representative left ventricular (LV) M-mode echocardiographic recordings. Daunorubicin administration significantly increased LV dimensions and reduced \% FS $(P<0.05)$, relative to the control group. With fenofibrate treatment, the increase in LV dimensions and reduction of \% FS were not statistically significant (Table 1, Fig. 5A and 5B).

To investigate whether adiponectin acts directly on cardiac tissues, we performed immunohistochemistry by staining heart sections with anti-adiponectin antibody. The presence of adiponectin proteins was observed in the representative rat myocardium specimens through immunohistochemistry study (Fig. 5C). The myocardial tissues obtained from the rats in the HF group expressed significantly higher amount of adiponectin, compared to the myocardium obtained from the rats in the fenofibrate group and normal controls. 
A
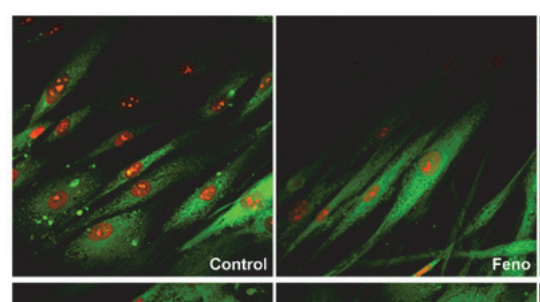

B

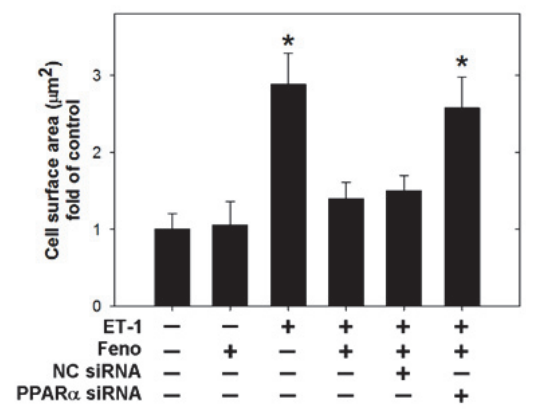

Fig.4. Cell hypertrophy of cardiomyocytes induced by endothelin-1 (ET-1) was inhibited by pre-treatment with fenofibrate. (A) To assess cellular hypertrophy, the cell size of cardiomyocytes was observed using Confocal Immunofluorescence study. ET-1 significantly increased the cell size of cardiomyocytes (upper right panel), compared to that of the control group (upper left panel); however, fenofibrate inhibited ET-1-induced cellular hypertrophy (lower left panel). In addition, the small interfering RNA (siRNA) of peroxisome proliferator-activated receptor $\alpha$ $(\operatorname{PPAR} \alpha$ ), instead of Negative Contral (NC) siRNA, abolished the effects of fenofibrate (lower middle and right panels). (B) Quantitative results of the cell surface area of cardiomyocytes treated with fenofibrate, PPAR $\alpha$ siRNA, or NC siRNA in response to ET-1 for 48 hours ( $n \geqq 10$ cells per experimental group). Results are representative of three independent experiments.

\section{Discussion}

The study demonstrated that in the cultured HCM, ET-1 caused a significant increase in cardiomyocyte size, adiponectin protein expression, and down-regulation of PPAR $\alpha$; however, such increase of cell size and enhancement of adiponectin expression were reduced by pre-treatment with fenofibrate. The addition of siRNA of PPAR $\alpha$ abolished the effects of fenofibrate, suggesting that the beneficial effects of fenofibrate on cardiomyocytes are exerted through PPAR $\alpha$ modulation. Moreover, in a rat model of daunorubicin-induced cardiomyopathy, fenofibrate treatment improved left ventricular contraction, reduced the elevated circulating adiponectin levels, and ameliorated adiponectin protein expression in the myocardium.

ET-1 plays a critical role in the progression of myocyte hypertrophy ${ }^{2}$. The influence of ET-1 on the renin-angiotensin-aldosterone system also plays an important role in the structural, electrical, and neurohormonal remodeling of myocardium ${ }^{26)}$. Down-regulation of PPAR $\alpha$ is observed in diseased hearts, such as those with cardiac hypertrophy and failure ${ }^{3-6}$. ET-1 caused a significant down-regulation of PPAR $\alpha$ in cultured HCM as observed in our study, and this is a novel finding and provides a strong support to the notion that PPAR $\alpha$ deficiency can lead to impaired functional capacity of the heart ${ }^{8}$. PPAR $\alpha$ activator fenofibrate reduces cardiac hypertrophy, decreases cardiac fibrosis, attenuates cardiac dysfunction and improves survival, and causes suppression of proinflammatory, prohypertrophic, and profibrotic gene expression $^{7-10,13,14)}$. Animal study has clearly demonstrated that myocardial fibrosis can be effectively inhibited by fenofibrate through suppression of AP-1mediated ET-1 gene augmentation ${ }^{8)}$. Our data also confirm that ET-1-induced cell hypertrophy and the decrease in the PPAR $\alpha$ level in cardiomyocytes can be improved by fenofibrate, which is consistent with previous reports.

Another important and intriguing finding of our study is that ET-1 time- and dose-dependently enhanced the expression of adiponectin; whereas, such an enhancement of adiponectin expression was inhibited by pre-treatment with fenofibrate. Moreover, addition of siRNA of PPAR $\alpha$ abolished the effects of fenofibrate. Recently, ET-1 has been shown as a regulatory factor in the secretion of different adipokines, including adiponectin ${ }^{27)}$. Through the endothelin type A receptor, ET-1 stimulates adipocytes to secrete adiponectin, suggesting that ET-1 may play a significant role in the regulation of adiponectin in adipose tissue $^{27)}$. The reasons and detailed mechanisms as to why 
Table 1. Demographic, echocardiographic data and circulating biomarkers of the rats in the in vivo study

\begin{tabular}{lccc}
\hline \multicolumn{1}{c}{ Parameters } & $\begin{array}{c}\text { Control group } \\
(\mathrm{N}=6)\end{array}$ & $\begin{array}{c}\text { HF group } \\
(\mathrm{N}=6)\end{array}$ & $\begin{array}{c}\text { FENO group } \\
(\mathrm{N}=6)\end{array}$ \\
\hline Body weight (g) 5 week/17 week & $160.5 \pm 0.5 / 280.3 \pm 21.6$ & $156.5 \pm 1.5 / 331.7 \pm 17.5^{*}$ & $158.5 \pm 0.7 / 352.0 \pm 16.5^{*}$ \\
Heart weight (g) & $0.76 \pm 0.25$ & $1.01 \pm 0.10^{*}$ & $0.98 \pm 0.11^{*}$ \\
HW/BW (\%) & $0.27 \pm 0.07$ & $0.30 \pm 0.02^{*}$ & $0.28 \pm 0.03$ \\
Adiponectin (ng/mL) & $3.36 \pm 2.5$ & $11.3 \pm 7.8^{*}$ & $6.11 \pm 5.5^{*} * *$ \\
Baseline FS (\%) & $62 \pm 3$ & $59 \pm 3$ & $61 \pm 2$ \\
FS at 12-week (\%) & $59 \pm 3$ & $52 \pm 3^{*}$ & $57 \pm 2$ \\
\hline
\end{tabular}

${ }^{*} P<0.05$ vs. Control group; ${ }^{* *} P<0.05$ vs. HF group; $(\mathrm{N})$ is the number of animals used to calculate the mean \pm S.E.M. of the presented data in Control group (normal control rats), HF group (rats received daunorubicin followed by treatment with vehicle), and FENO group (rats received daunorubicin followed by treatment with fenofibrate).

HF, heart failure; FENO, fenofibrate; HW, heart weight; BW, body weight; FS, fractional shortening

\section{Control}

A

Baseline Echo

B

12 weeks Echo

C

12 weeks IHC

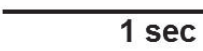

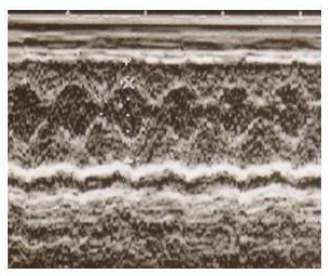

HF
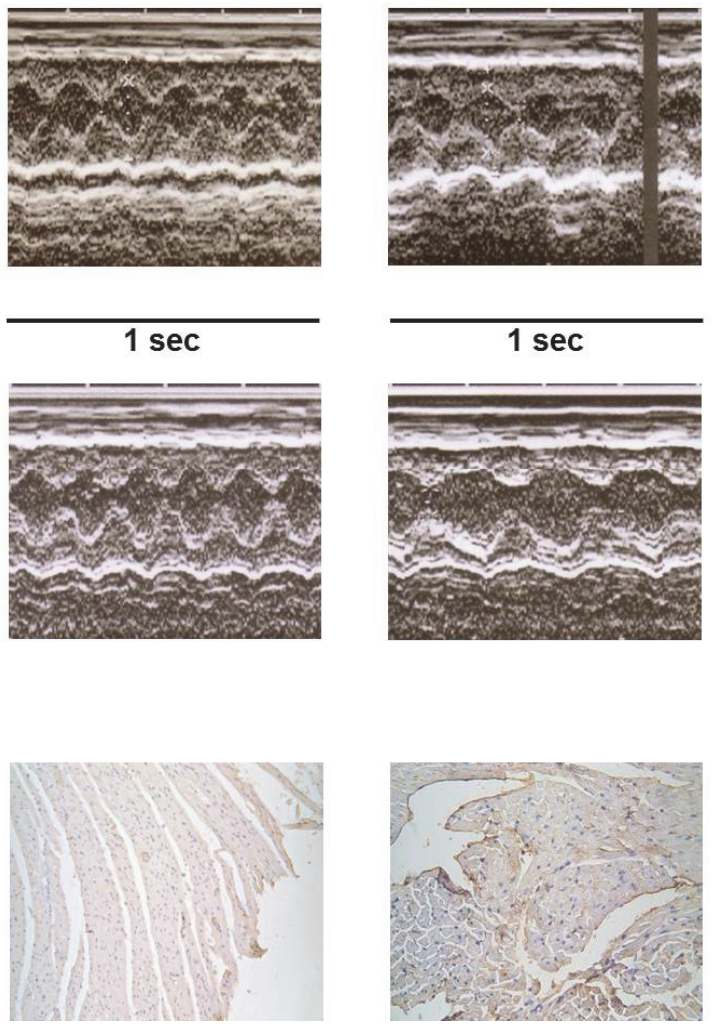

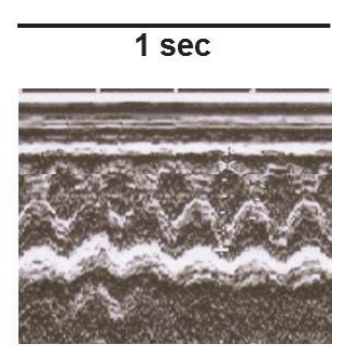

FENO
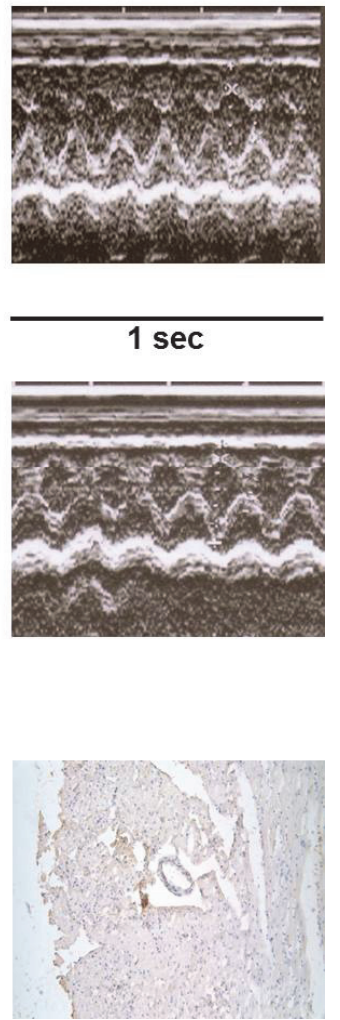

Fig. 5. In the rat model of daunorubicin-induced cardiomyopathy, panels A and B show representative left ventricular (LV) M-mode echocardiographic recordings at baseline and at 12th week, respectively. Daunorubicin administration significantly increased LV dimensions (HF) and the increase in LV dimensions was not statistically significant with the treatment of fenofibrate. C. Immunohistochemistry study (IHC) demonstrates that the myocardial expression of adiponectin is significantly higher in the HF group, compared to those in the myocardium obtained from the rats in the fenofibrate group and normal controls.

adiponectin expression increases in response to ET-1 stimulation in cultured HCM remain unclear. One possible explanation is that the elevated adiponectin is a physiological counter-regulating response to ET-1induced downregulation of PPAR $\alpha$. One recent study has demonstrated that adiponectin significantly 
enhanced $\operatorname{PPAR} \alpha$ activity, and the adiponectin-dependent PPAR $\alpha$ activation played a protective role against angiotensin II-induced cardiac fibrosis ${ }^{19)}$. The other possible explanation is that acutely enhanced expression of adiponectin to ET-1 stimulation or accumulation of adiponectin in injured heart tissues may represent a compensatory response, and probably acts as an endogenous anti-hypertrophic and anti-fibrotic agent, which leads to protection against the progression of myocardial injury through regulation of cardiac remodeling ${ }^{17,18)}$, much like natriuretic peptides. Through activation of AMP-activated protein kinase (AMPK) signaling, adiponectin inhibits hypertrophic signaling in the myocardium, including that induced by endothelin stimulation ${ }^{17,18,20,28)}$. Moreover, supplementation of exogenous adiponectin may directly protect cardiomyocytes in adiponectin-deficient animals ${ }^{17,18,28)}$.

However, adiponectin is best known to confer numerous cardioprotective effects, but it would be naive to assume that this has always been the case. Plasma adiponectin concentrations are increased in patients with overt heart failure and high adiponectin levels are independently predictive of clinical outcomes in heart failure ${ }^{29,30)}$. In our previous study, the myocardial tissue expression of adiponectin was related to the severity of heart failure, suggesting that adiponectin may play a role in the progression of heart failure and metabolism disturbance in advanced heart failure $^{30)}$. In fact, some of the effects of adiponectin may adversely affect the cardiovascular system or even the whole body while being chronically activated, such as in the case of chronic heart failure. For instance, adiponectin may activate $\mathrm{NF}-\kappa \mathrm{B}$ and $\mathrm{AP}-1$, resulting in expression of proinflammatory genes and enhancement of angiotensin $I I$-induced proliferation in cardiac fibroblasts, which may in turn play a role in heart failure progression ${ }^{31,32)}$. Moreover, adiponectin can increase energy expenditure and decrease body weight, which may participate in the development of cardiac cachexia $^{33,34)}$. Therefore, the increase in plasma adiponectin in chronic heart failure can also represent shortterm beneficial adaptive responses on acute injury, which may eventually turn into harmful maladaptive signals on prolonged and chronic activation, like the sustained sympathetic hyperactivity in the advanced heart failure ${ }^{35)}$. In actuality, greater cardiac disease severity can lead to greater salvage attempts; hence, a higher adiponectin concentration. If so, with successful anti-failure therapy, the plasma adiponectin concentrations should return to baseline conditions with improvement of HF. Supporters of this hypothesis have recently published reports to demonstrate that anti-failure treatment was associated with reduced plasma adiponectin concentration and the improve- ment of left ventricular ejection fraction in both acute and chronic heart failure patients ${ }^{35,36)}$. In our in vivo study, that also supports this hypothesis, we have discovered that fenofibrate treatment is able to significantly reduce circulating levels of adiponectin, improve the left ventricular function, and reverse the myocardial expression of adiponectin. However, whether the use of fenofibrate really works in the prevention of cardiac hypertrophy or treatment of heart failure patients, still awaits confirmation via further clinical trials. Whether adipokines, such as adiponectin, may become potential targets for the treatment, also calls for further investigations.

Our study does not exclude a role for the effects of PPAR $\alpha$ activators on the expression of other factors involved in cardiomyocyte hypertrophy and adiponectin expression. Nor does it rule out the possibility that the molecules tested may act through unrelated mechanisms in addition to PPAR $\alpha$ activation. For instance, fenofibrate can suppress ET-1-induced cardiac hypertrophy by down-regulation of AP-1 binding capability and inhibition of $\mathrm{p} 38$ signaling ${ }^{11,12}$. In our previous study, we demonstrated that angiotensin II-enhanced adiponectin expression in cultured HCM can be inhibited by angiotensin receptor blocker through ERK, $\mathrm{p} 38$, and JNK pathways ${ }^{22)}$. Therefore, it is reasonable to hypothesize that such a link may also exist between various mitogen-activated protein kinase signaling cascades, PPAR $\alpha$, adiponectin, and cardiac hypertrophy. However, it is only fair to emphasize here that the present study was designed to merely study the possible physiologic protective role of fenofibrate; hence, the study results proffer some clues to the research of the mechanisms of adiponectin in the pathophysiology of cardiac hypertrophy. No definite conclusion can be drawn regarding which mechanisms are more relevant from the present study. Further investigation into the pathophysiologic interaction among adiponectin, adiponectin receptors, together with their interplay in signal transduction, so as to gain additional insight into the precise regulatory mechanisms for the myocardial expression of adiponectin in cardiomyocyte hypertrophy and the complex interaction between ET-1, adiponectin, PPAR $\alpha$, and fenofibrate, will be most fascinating and has the potential to lead to the discovery of novel treatment strategies for cardiac hypertrophy and heart failure.

\section{Conclusions}

In conclusion, we have demonstrated that adiponectin, ET-1, and PPAR $\alpha$ are closely associated with one another and partly contribute to the pathogenesis of cardiac hypertrophy and heart failure, but the pre- 
cise mechanisms are not yet fully clear. However, this study may proffer some clues to the research of the mechanisms of adiponectin in the pathophysiology of cardiac hypertrophy and heart failure. Further clinical studies on a larger scale are also required to confirm whether fenofibrate may protect the heart against acute and chronic injuries.

\section{Acknowledgments}

The research team is most grateful for the generous subsidies provided by the Research Grant of Cheng Hsin General Hospital-National Yang-Ming University Cooperation Project (no. 98F117CY09) and Research Grant of Cheng Hsin General Hospital (no. 99-15). We also thank Ms. Chin-Feng Cheng for her excellent assistance in preparing and typing the manuscript.

\section{Conflict of Interest}

The authors declare that there is no conflict of interest regarding the publication of this paper.

\section{References}

1) Balakumar P, Jagadeesh G: Multifarious molecular signaling cascades of cardiac hypertrophy: can the muddy waters be cleared? Pharmacol Res, 2010; 62: 365-383

2) Drawnel FM, Archer CR, Roderick HL: The role of the paracrine/autocrine mediator endothelin-1 in regulation of cardiac contractility and growth. Br J Pharmacol, 2013; 168: 296-317

3) Schiffrin EL: Peroxisome proliferator-activated receptors and cardiovascular remodeling. Am J Physiol Heart Circ Physiol, 2005; 288: H1037-H1043

4) Smeets PJ, de Vogel-van den Bosch HM, Willemsen PH, Stassen AP, Ayoubi T, van der Vusse GJ, van Bilsen M: Transcriptomic analysis of PPARalpha-dependent alterations during cardiac hypertrophy. Physiol Genomics, 2008; 36: 15-23

5) Smeets PJ, Teunissen BE, Willemsen PH, van Nieuwenhoven FA, Brouns AE, Janssen BJ, Cleutjens JP, Staels B, van der Vusse GJ, van Bilsen M: Cardiac hypertrophy is enhanced in PPAR alpha-/- mice in response to chronic pressure overload. Cardiovasc Res, 2008; 78: 79-89

6) Oyekan A: PPARs and their effects on the cardiovascular system. Clin Exp Hypertens, 2011; 33: 287-293

7) Labinskyy V, Bellomo M, Chandler MP, Young ME, Lionetti V, Qanud K, Bigazzi F, Sampietro T, Stanley WC, Recchia FA: Chronic activation of peroxisome proliferator-activated receptor-alpha with fenofibrate prevents alterations in cardiac metabolic phenotype without changing the onset of decompensation in pacing-induced heart failure. J Pharmacol Exp Ther, 2007; 321: 165-171

8) Ogata T, Miyauchi T, Sakai S, Irukayama-Tomobe Y, Goto K, Yamaguchi I: Stimulation of peroxisome-proliferator-activated receptor alpha (PPAR alpha) attenuates cardiac fibrosis and endothelin-1 production in pressureoverloaded rat hearts. Clin Sci (Lond), 2002; 103 (Suppl 48): $284 \mathrm{~S}-288 \mathrm{~S}$

9) Ichihara S, Obata K, Yamada Y, Nagata K, Noda A, Ichihara G, Yamada A, Kato T, Izawa H, Murohara T, Yokota M: Attenuation of cardiac dysfunction by a PPAR-alpha agonist is associated with down-regulation of redox-regulated transcription factors. J Mol Cell Cardiol, 2006; 41: 318-329

10) Huang WP, Yin WH, Chen JW, Jen HL, Young MS, Lin SJ: Fenofibrate attenuates endothelial monocyte adhesion in chronic heart failure: an in vitro study. Eur J Clin Invest, 2009; 39: 775-783

11) Irukayama-Tomobe $Y$, Miyauchi T, Sakai S, Kasuya Y, Ogata T, Takanashi M, Iemitsu M, Sudo T, Goto K, Yamaguchi I: Endothelin-1-induced cardiac hypertrophy is inhibited by activation of peroxisome proliferator-activated receptor-alpha partly via blockade of c-Jun NH2terminal kinase pathway. Circulation, 2004; 109: 904-910

12) Irukayama-Tomobe $Y$, Miyauchi T, Kasuya Y, Sakai S, Goto K, Yamaguchi I: Activation of peroxisome proliferator-activated receptor-alpha decreases endothelin-1-induced p38 mitogen-activated protein kinase activation in cardiomyocytes. J Cardiovasc Pharmacol, 2004; 44 (Suppl 1): S358-S361

13) Le K, Li R, Xu S, Wu X, Huang H, Bao Y, Cai Y, Lan T, Moss J, Li C, Zou J, Shen X, Liu P: PPAR $\alpha$ activation inhibits endothelin-1-induced cardiomyocyte hypertrophy by prevention of NFATc4 binding to GATA-4. Arch Biochem Biophys, 2012; 518: 71-78

14) Zou J, Le K, Xu S, Chen J, Liu Z, Chao X, Geng B, Luo J, Zeng S, Ye J, Liu P: Fenofibrate ameliorates cardiac hypertrophy by activation of peroxisome proliferator-activated receptor- $\alpha$ partly via preventing $\mathrm{p} 65-\mathrm{NF} \kappa \mathrm{B}$ binding to NFATc4. Mol Cell Endocrinol, 2013; 370: 103-112

15) Planavila A, Laguna JC, Vázquez-Carrera M: Atorvastatin improves peroxisome proliferator-activated receptor signaling in cardiac hypertrophy by preventing nuclear factor-kappa B activation. Biochim Biophys Acta, 2005; 1687: 76-83

16) Park M, Sweeney G: Direct effects of adipokines on the heart: focus on adiponectin. Heart Fail Rev, 2013; 18: 631-644

17) Goldstein BJ, Scalia RG, Ma XL: Protective vascular and myocardial effects of adiponectin. Nat Clin Pract Cardiovasc Med, 2009; 6: 27-35

18) Shibata R, Ouchi N, Ito M, Kihara S, Shiojima I, Pimentel DR, Kumada M, Sato K, Schiekofer S, Ohashi K, Funahashi T, Colucci WS, Walsh K: Adiponectin-mediated modulation of hypertrophic signals in the heart. Nat Med, 2004; 10: 1384-1389

19) Fujita K, Maeda N, Sonoda M, Ohashi K, Hibuse T, Nishizawa H, Nishida M, Hiuge A, Kurata A, Kihara S, Shimomura I, Funahashi T: Adiponectin protects against angiotensin $I I$-induced cardiac fibrosis through activation of PPAR-alpha. Arterioscler Thromb Vasc Biol, 2008; 28: 863-870

20) Fujioka D, Kawabata K, Saito Y, Kobayashi T, Nakamura T, Kodama Y, Takano H, Obata JE, Kitta Y, Umetani K, Kugiyama K: Role of adiponectin receptors in endothelininduced cellular hypertrophy in cultured cardiomyocytes 
and their expression in infarcted heart. Am J Physiol Heart Circ Physiol, 2006; 290: H2409-H2416

21) Yin WH, Chen YH, Wei J, Jen HL, Huang WP, Young MS, Chen DC, Liu PL: Associations between endothelin-1 and adiponectin in chronic heart failure. Cardiology, 2011; 118: 207-216

22) Yin WH, Chen JW, Lin SJ, Young MS: Effect of irbesar$\tan$ on angiotensin II-induced adiponectin expression in human cardiomyocytes. Acta Cardiologica Sinica, 2011: 27: 244-251

23) Watkins SJ, Borthwick GM, Oakenfull R, Robson A, Arthur HM: Angiotensin II-induced cardiomyocyte hypertrophy in vitro is TAK1-dependent and Smad2/3independent. Hypertens Res, 2012; 35: 393-398

24) Balfour JA, McTavish D, Heel RC: Fenofibrate. A review of its pharmacodynamic and pharmacokinetic properties and therapeutic use in dyslipidaemia. Drugs, 1990; 40: 260-290

25) Soga M, Kamal FA, Watanabe K, Ma M, Palaniyandi S, Prakash P, Veeraveedu P, Mito S, Kunisaki M, Tachikawa H, Kodama M, Aizawa Y: Effects of angiotensin II receptor blocker (candesartan) in daunorubicin-induced cardiomyopathic rats. Int J Cardiol, 2006; 10: 378-385

26) Takahashi T, Saegusa S, Sumino H, Nakahashi T, Iwai K, Morimoto S, Nojima T, Kanda T: Adiponectin, T-cadherin and tumour necrosis factor-alpha in damaged cardiomyocytes from autopsy specimens. J Int Med Res, 2005; 33: 236-244

27) Clarke KJ, Zhong Q, Schwartz DD, Coleman ES, Kemppainen RJ, Judd RL: Regulation of adiponectin secretion by endothelin-1. Biochem Biophys Res Commun, 2003; 312: 945-949

28) Kistorp C, Faber J, Galatius S, Gustafsson F, Frystyk J, Flyvbjerg A, Hildebrandt P: Plasma adiponectin, body mass index, and mortality in patients with chronic heart failure. Circulation, 2005; 112: 1756-1762

29) Yin WH, Wei J, Huang WP, Chen JW, Young MS, Lin
SJ: Prognostic value of circulating adipokine levels and expressions of adipokines in the myocardium of patients with chronic heart failure. Circ J, 2012; 76: 2139-2147

30) Hattori Y, Hattori S, Kasai K: Globular adiponectin activates nuclear factor-kappaB in vascular endothelial cells, which in turn induces expression of proinflammatory and adhesion molecule genes. Diabetes Care, 2006; 29: 139141

31) Hattori $Y$, Hattori S, Akimoto K, Nishikimi T, Suzuki K, Matsuoka H, Kasai K: Globular adiponectin activates nuclear factor-kappaB and activating protein-1 and enhances angiotensin II-induced proliferation in cardiac fibroblasts. Diabetes, 2007; 56: 804-808

32) Fruebis J, Tsao TS, Javorschi S, Ebbets-Reed D, Erickson MR, Yen FT, Bihain BE, Lodish HF: Proteolytic cleavage product of $30-\mathrm{kDa}$ adipocyte complement-related protein increases fatty acid oxidation in muscle and causes weight loss in mice. Proc Natl Acad Sci U S A, 2001; 98: 20052010

33) Qi Y, Takahashi N, Hileman SM, Patel HR, Berg AH, Pajvani UB, Scherer PE, Ahima RS: Adiponectin acts in the brain to decrease body weight. Nat Med, 2004; 10: 524-529

34) Araújo JP, Lourenço P, Rocha-Gonçalves F, Ferreira A, Bettencourt P: Adiponectin is increased in cardiac cachexia irrespective of body mass index. Eur J Heart Fail, 2009; 11: 567-572

35) Yamaji M, Tsutamoto T, Tanaka T, Kawahara C, Nishiyama K, Yamamoto T, Fujii M, Horie M: Effect of carvedilol on plasma adiponectin concentration in patients with chronic heart failure. Circ J, 2009; 73: 1067-1073

36) Matsumoto $M$, Lee-Kawabata $M$, Tsujino T, Naito $Y$, Ezumi A, Sakoda T, Ohyanagi M, Shimomura I, Masuyama T: Decrease in serum adiponectin levels in response to treatment predicts good prognosis in acute decompensated heart failure. J Clin Hypertens (Greenwich), 2010; 12: 900-904 